research support from: Celgene, Frances Humby: None declared, Costantino Pitzalis Grant/research support from: Celgene DOI: 10.1136/annrheumdis-2019-eular.3634

\section{OP0114 MACHINE LEARNING TOOLS IDENTIFY PATIENT CLUSTERS AND SWOLLEN AND TENDER JOINT CORRELATION PATTERNS IN A LARGE DATABASE FROM THE SECUKINUMAB PSORIATIC ARTHRITIS CLINICAL DEVELOPMENT PROGRAM}

Matthias Kormaksson ${ }^{1}$, Effie Pournara ${ }^{2}$, Gregory Ligozio ${ }^{1}$, Luminita Pricop ${ }^{1}$, Ken Abrams ${ }^{1}$, Bruce Kirkham ${ }^{3}$, Kristian Reich ${ }^{4}$, lain McInnes ${ }^{5} .{ }^{1}$ Novartis Pharmaceutical Corporation, New Jersey, United States of America; ${ }^{2}$ Novartis Pharma AG, Basel, Switzerland; ${ }^{3}$ Guy's and St Thomas' NHS Foundation Trust, London, United Kingdom; ${ }^{4}$ University Medical Center Hamburg-Eppendorf, Skin Inflammation Center, Hamburg, and Dermatologikum, Berlin, Germany, ${ }^{5}$ University of Glasgow, Glasgow, United Kingdom

Background: Identifying patient phenotypes using machine-learning (ML) techniques amidst the variability and heterogeneity of the clinical manifestations of psoriatic arthritis (PsA) could be the first critical step towards better understanding of the disease eventually leading to individualized medicine.

Objectives: To identify distinct clusters of patients with PsA based on patients tender joint (TJ) and swollen joint (SJ) counts and correlation patterns among $T J$ and SJ counts at baseline as captured in the secukinumab FUTURE trials program.

Methods: Pairwise correlations were explored among 76 SJ and 78 TJ measurements of $>2,700$ patients with PsA across 5 phase III studies with $\approx 425,000$ data entries at baseline and were visualized using heatmaps. Due to high correlations between SJs and corresponding TJs, a composite variable "swollen/tender joint count" was constructed for each joint. Hierarchical clustering was then performed on the composite using "1-correlation" as the dissimilarity metric and Ward's agglomeration method for pairwise grouping of joints. A dendrogram was used to visualize and assess the resulting joint groupings.

Results: The hierarchical clustering algorithm grouped the 78 individual joints into distinct and natural clusters (Figure 1A). At higher level of the dendrogram, the algorithm grouped separately all foot, larger (jaws, clavicles, ankles, hips, wrists, knees, shoulders, elbows), and hand joints. Cutting the dendrogram at 15 clusters separated all the joints into distinct groups; hand joints (distal and proximal phalanges, metacarpals and thumbs), and foot joints (distal and proximal phalanges, metatarsals and big toes). Similar clustering algorithms were explored to identify patient clusters at baseline with distinct swelling and tenderness patterns across the identified joint groups. High correlation between swelling/tenderness of the left and swelling/tenderness of the corresponding right joint was observed across all individual joints (Figure 1B); a high correlation was also observed between swelling and tenderness at all individual joints. More localized patterns showed that there is a gradual decrease in correlation (from highest to lowest) among TJs and SJs in adjacent vs non-adjacent fingers, which is evident from

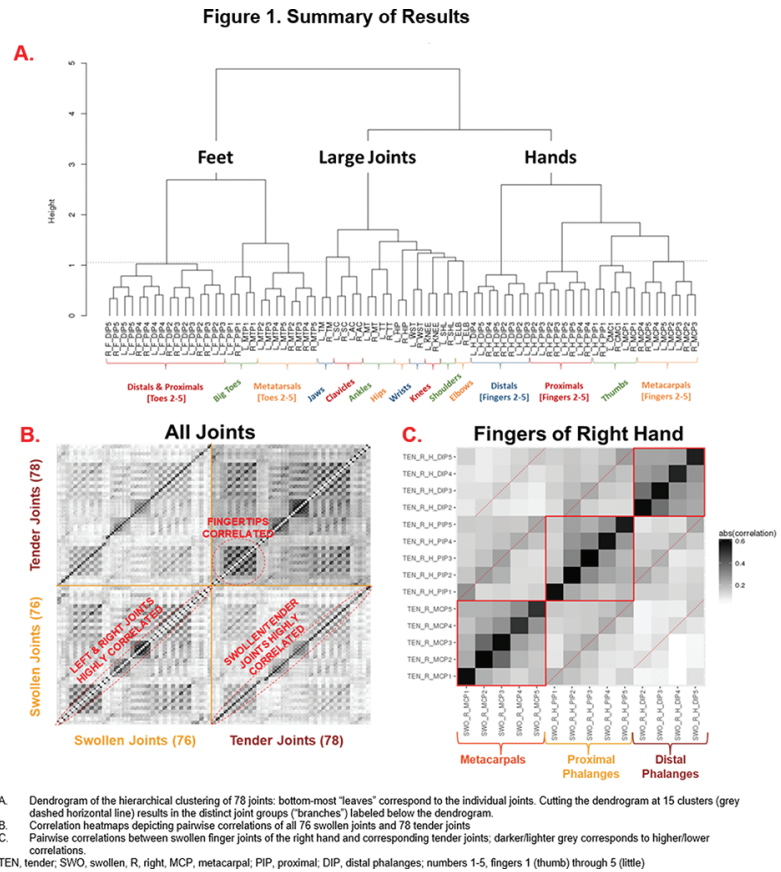

grey-scale patterns (Figure 1C). Specifically, a gradual decrease in correlation between the swelling of $2^{\text {nd }}$ distal interphalangeal joint and the tenderness of the $2^{\text {nd }}-5^{\text {th }}$ distal phalanges was noted.

Conclusion: Machine learning methodology confirmed a natural grouping of joints in patients with psoriatic arthritis based on baseline swelling and tenderness and revealed complex correlation patterns. Additional cluster analyses have demonstrated distinct patient clusters across the identified joint groups. Further inves tigating potential associations of other disease manifestations such as skin and nail involvement to define additional phenotypes may explain differences in disease pathogenesis and treatment outcomes.

\section{REFERENCE:}

[1] Grys BT, et al., J Cell Biol. 2017; 216(1): 65-71.

Disclosure of Interests: Matthias Kormaksson Shareholder of: Novartis, Employee of: Novartis, Effie Pournara Shareholder of: Novartis, Employee of Novartis, Gregory Ligozio Shareholder of: Novartis, Employee of: Novartis, Luminita Pricop Shareholder of: Novartis, Employee of: Novartis, Ken Abrams Shareholder of: Novartis, Employee of: Novartis, Bruce Kirkham Grant/research support from: Abbvie, Janssen, Lilly, Novartis, Roche, UCB, Consultant for: Abbvie, Jans sen, Lilly, Novartis, Roche, UCB, Speakers bureau: Abbvie, Janssen, Lilly, Novartis, Roche, UCB, Kristian Reich Consultant for: Abbvie, Affibody, Amgen, Biogen, Boehringer Ingelheim Pharma, Celgene, Centocor, Covagen, Forward Pharma Fresenius Medical Care, GlaxoSmithKline, Janssen-Cilag, Kyowa Kirin, Leo, Lilly, Medac, Merck Sharp \& Dohme Corp., Novartis, Miltenyi Biotec, Ocean Pharma Pfizer, Regeneron, Samsung Bioepis, Sanofi, Takeda, UCB Pharma, Valeant, Xenoport; Speakers bureau: Abbvie, Affibody, Amgen, Biogen, Boehringer Ingelheim Pharma, Celgene, Centocor, Covagen, Forward Pharma, Fresenius Medical Care, GlaxoSmithKline, Janssen-Cilag, Kyowa Kirin, Leo, Lilly, Medac, Merck Sharp \& Dohme Corp., Novartis, Miltenyi Biotec, Ocean Pharma, Pfizer, Regeneron, Samsung Bioepis, Sanofi, Takeda, UCB Pharma, Valeant, Xenoport, lain Mclnnes Grant/research support from: AstraZeneca, Celgene, Compugen, Novartis, Roche, UCB Pharma, Consultant for: AbbVie, Celgene, Galvani, Lilly, Novartis, Pfizer, UCB Pharma

DOI: 10.1136/annrheumdis-2019-eular.1910

\section{OP0115 \\ GENERAL AND SEX-SPECIFIC PREDICTORS OF PSA AMONG PATIENTS WITH PSORIASIS}

Alexis Ogdie ${ }^{1}$, Jose Scher ${ }^{2}$, Shiyu Vanessa Wang ${ }^{1}$, Daniel Shin ${ }^{1}$, David Margolis ${ }^{1}$, Junko Takeshita ${ }^{1}$, Hyon $\mathrm{Choi}^{3}$, Thorvardur Jon Love ${ }^{4}$, Christopher T. Ritchlin ${ }^{5}$, Joel Gelfand ${ }^{1}$, Joseph F. Merola ${ }^{6} .{ }^{1}$ University of Pennsylvania, Philadelphia, PA, United States of America; ${ }^{2}$ New York University, New York, NY, United States of America; ${ }^{3}$ Massachusetts General Hospital, Boston, MA, United States of America; ${ }^{4}$ University of Iceland, Reykjavík, Iceland; ${ }^{5}$ University of Rochester, Rochester, NY, United States of America; ${ }^{6}$ Brigham Women's Hospital, Boston, MA, United States of America

Background: Risk prediction models in electronic health record (EHR) databases may assist in early identification of patients with psoriasis likely to develop psoriatic arthritis (PsA). ${ }^{1}$ A better understanding of potential predictors and whether stratification by sex would be needed in building such algorithms is required. ${ }^{1}$

Objectives: Examine general and sex-specific predictors of PsA in an EHR dataset among patients with psoriasis

Methods: A retrospective cohort study was performed within the Optumlnsights EHR Database (United States) between 2006-2017. Patients with two or more ICD codes for psoriasis and ages 16-90 were identified. The outcome was PsA (defined by a single ICD code). Potential predictors, in particular comorbidities and infections, were also identified using ICD codes. Hazard ratios were calculated using Cox proportional hazards models between individual predictors and development of incident PsA in univariate models and those that were significant $(p<0.1)$ were entered into a multivariable model. A final model was achieved using automated stepwise regression. Separate models were developed for each sex as some predictors (e.g., polycystic ovarian syndrome, prostatitis) are sex-specific.

Results: Among 215,386 patients with psoriasis, mean age was 50 (SD 15.6) and $55 \%$ were female. At index date (one year after date of first psoriasis code), $4.6 \%$ and $4.2 \%$ of patients had been prescribed a biologic therapy or oral therapy in the past year. Mean follow up time was 5.6 years (SD 2.8) and 4,288 patients developed incident PsA (incidence 3.5 cases/1,000 person years). Previously identified predictors were significant in univariate models (depression, fatigue, inflammatory bowel disease, uveitis, hyperlipidemia, fracture; data not shown due to space restrictions) but several new predictors were also identified (diabetes, hidradenitis suppurativa, celiac disease, irritable bowel syndrome, sepsis, post-traumatic stress disorder, anxiety, anemia) (Table). Automated regression identified subsets of these factors in multivariable models; these models differed by sex.

Conclusion: Predictors of developing PsA differed by sex but obesity, depression, and fatigue were statistically significant predictors in both groups. Infections were also associated with development of PsA but the type of infection differed by sex. 
REFERENCES:

[1] Scher, et al. Nat Rev Rheum 2019 In Press.

Table. Multivariable HRs for the risk for PsA among patients with psoriasis.

\begin{tabular}{lccc}
\hline & All $^{*}$ & Women & Men \\
\hline Age & $0.99(0.99-1.00)$ & $1.00(0.99-1.00)$ & $0.99(0.99-0.99)$ \\
Male Sex & $1.09(1.02-1.16)$ & & \\
Obesity & $1.31(1.16-1.48)$ & $1.30(1.11-1.53)$ & $1.35(1.12-1.64)$ \\
Depression & $1.19(1.06-1.33)$ & $1.19(1.04-1.37)$ & $1.23(1.01-1.49)$ \\
Fatigue & $1.61(1.43-1.81)$ & $1.50(1.30-1.75)$ & $1.91(1.59-2.29)$ \\
Anemia & $1.48(1.29-1.70)$ & $1.62(1.37-1.92)$ & \\
Uveitis & $2.48(1.41-4.38)$ & $2.90(1.38-6.08)$ & \\
Sepsis & $1.64(1.07-2.52)$ & $2.39(1.41-4.03)$ & \\
Liver Disease & $1.31(1.06-1.62)$ & & $1.40(1.04-1.88)$ \\
Hiddradenitis Suppertiva & $2.16(1.16-4.02)$ & & $4.04(1.68-9.74)$ \\
Hypertension & $1.16(1.07-1.26)$ & & $1.18(1.05-1.33)$ \\
Osteomyelitis & $2.17(1.29-3.67)$ & & \\
Celiac Disease & $1.98(1.10-3.58)$ & & \\
HIV & $0.24(0.06-0.96)$ & & \\
Any infection & $1.13(1.04-1.22)$ & & \\
Restless Leg Syndrome & & $1.55(1.06-2.28)$ & \\
Salmonella & & $1.38-5.29)$ \\
Cellulitis & & $1.23(1.06-1.43)$ & \\
Diabetes & & & $1.62(1.00-2.62)$ \\
Irritable Bowel Syndrome & & & $4.40(1.10-17.62)$ \\
Venous Thromboembolism & & &
\end{tabular}

Disclosure of Interests: Alexis Ogdie Grant/research support from: (To my university) Novartis, Pfizer, Grant/research support from: Novartis, Pfizer, Grant/ research support from: Novartis, Pfizer, Grant/research support from: Novartis, Pfizer, Consultant for: AbbVie, Bristol-Myers Squibb, Celgene, Corrona, Eli Lilly and Company, Novartis, Pfizer, and Takeda, Consultant for: AbbVie, Amgen, Bristol-Myers Squibb, Celgene, Corrona, Eli Lilly, Novartis, Pfizer Inc, Takeda, Consultant for: Abbvie, Amgen, BMS, Celgene, Corrona, Lilly, Novartis, Pfizer, Takeda, Consultant for: Abbvie, Amgen, BMS, Celgene, Corrona, Lilly, Novartis, Pfizer, Takeda, Jose Scher Grant/research support from: Pfizer, Novartis, Consultant for: Janssen, UCB, Novartis, Amgen, Shiyu Vanessa Wang: None declared, Daniel Shin: None declared, David Margolis Grant/research support from: Research funds from Valeant to the trustees of the university of pennsylvania, Consultant for: Data monitoring boards for Johnson and Johnson, Junko Takeshita Grant/research support from: Pfizer (to the Trustees of the University of Pennsylvania), Paid instructor for: Continuing medical education work related to psoriasis that was supported indirectly by Eli Lilly., Hyon Choi: None declared, Thorvardur Jon Love Consultant for: Received reimbursment from Celgene for speaking about guidelines for the treatment of psoriatic arthritis, Christopher $\mathrm{T}$. Ritchlin Grant/research support from: AbbVie, Amgen, UCB Pharma, Consultant for: AbbVie, Amgen, Lilly, Novartis, Pfizer, UCB Pharma, Joel Gelfand Grant/ research support from: Research grants (to the Trustees of the University of Pennsylvania) from Abbvie, Boehringer Ingelheim, Janssen, Novartis Corp, Celgene, Ortho Dermatologics, and Pfizer Inc., Consultant for: BMS, Boehringer Ingelheim, Janssen Biologics, Novartis Corp, UCB (DSMB), Sanofi, and Pfizer Inc., Paid instructor for: Received payment for continuing medical education work related to psoriasis that was supported indirectly by Lilly, Ortho Dermatologics and Novartis., Joseph F. Merola Consultant for: Biogen IDEC, Abbvie, Amgen, Eli Lilly and Company, Novartis, Pfizer, Janssen, UCB, Samumed, Celgene, Sanofi Regeneron, Merck, and GSK

DOI: 10.1136/annrheumdis-2019-eular.4390

THURSDAY, 13 JUNE 2019

"Rheumatoid arthritis - looking before, looking forward!"

\section{OP0116 JOINT EROSIONS VISIBLE ON ULTRASOUND PREDICT ARTHRITIS DEVELOPMENT IN PATIENTS WITH ACPA AND MUSCULOSKELETAL PAIN BUT NO SWOLLEN JOINTS}

Michael Ziegelasch ${ }^{1}$, Emma Eloff ${ }^{1}$, Hilde Berner Hammer ${ }^{2}$, Jan Cedergren ${ }^{1}$, Klara Martinsson ${ }^{1}$, Åsa Reckner ${ }^{1}$, Thomas Skogh ${ }^{1}$, Mattias Magnusson ${ }^{1}$, Alf Kastbom ${ }^{1}$. 'Linköping University, Dpt of Rheumatology and Dpt of Clinical and Experimental Medicine, Linköping, Sweden; ${ }^{2}$ Diakonhjemmet Hospital, Department of Rheumatology, Oslo, Norway

Background: Anti-citrullinated protein antibodies (ACPA) are associated with an increased risk of developing rheumatoid arthritis (RA), and in particular erosive disease. Detection of joint inflammation prior to clinical synovitis may improve treatment decisions in early disease.

Objectives: We sought to determine the value of ultrasound (US) to predict arthritis development among ACPA positive patients with musculoskeletal (MSK) pain. Methods: We prospectively followed 82 ACPA-positive patients with MSK pain but without arthritis upon baseline clinical examination (mean follow-up 68 months, range 23-91). US at baseline assessed joint erosions, synovial hypertrophy in grey scale (GS), and inflammatory activity judged by power Doppler (PD) in 36 small joints in hands and feet. We used a ProFocus system (BK Medical) with pulse repetition frequency $0.8 \mathrm{kHz}$ for $\mathrm{PD}$ grading. The US and $\mathrm{PD}$ results were blinded to patients and treating rheumatologists during the initial 3 years. US findings among patients were compared to 100 age-matched healthy blood donors Arthritis development during follow-up of patients was determined by clinical examination by an experienced rheumatologist. Associations between baseline US findings and arthritis development were tested by Cox regression analysis with adjustment for sex, age, symptom duration, smoking habits, erythrocyte sedimentation rate, C-reactive protein level, rheumatoid factor, and ACPA levels.

Results: Significantly more patient joints had synovial hypertrophy (GS score $>0$ ) compared to control joints in metacarpophalangeal (MCP) $(5.2 \%$ vs. $2.5 \%$ $\mathrm{p}<0.001$ ) and proximal interphalangeal (PIP) joints $2-5$ (6.6 vs. $1.5 \% ; \mathrm{p}<0.001)$. In contrast, metatarsophalangeal (MTP) joints $1-5$ of the controls were more often scored GS $>0$ compared to patient joints ( $49 \%$ vs. $24 \%$; $p<0.001)$. Positive PD $(>0)$ occurred significantly more often in patient joints compared to the controls in all joint areas $(p<0.05)$. At patient level, the mean sum score of all investigated joints was higher among patients than controls, regarding GS as well as PD $(\mathrm{p}<0.001$ for both). 13 patients $(16 \%)$, but none of the controls, had erosions detected on US $(p<0.001)$. During follow-up of patients, $39(48 \%)$ developed arthritis after median 25 weeks (range 5-302). Arthritis development was significantly more common among patients with baseline US erosions (10 out of 13 $77 \%$ ) compared to those without (29 out of $69 ; 42 \%, p=0.032$ ). This remained sig nificant also in Cox regression adjusting for potential confounders (Hazard ratio $=4.2,95 \% \mathrm{Cl} 1.7-10.4, \mathrm{p}=0.002)$. Out of the 13 erosions detected on US, 4 could be identified on conventional radiographs. Neither GS nor PD findings were significantly associated with arthritis development.

Conclusion: Arthritis-related US findings are more common among patients at increased risk of RA compared to healthy controls, but with site-specific differences. Erosions detected on US predicted arthritis development. Thus, US assess ment of erosions improves risk-stratification of ACPA-positive patients without swollen joints, and potentially identifies patients eligible for very early pharmacotherapy.

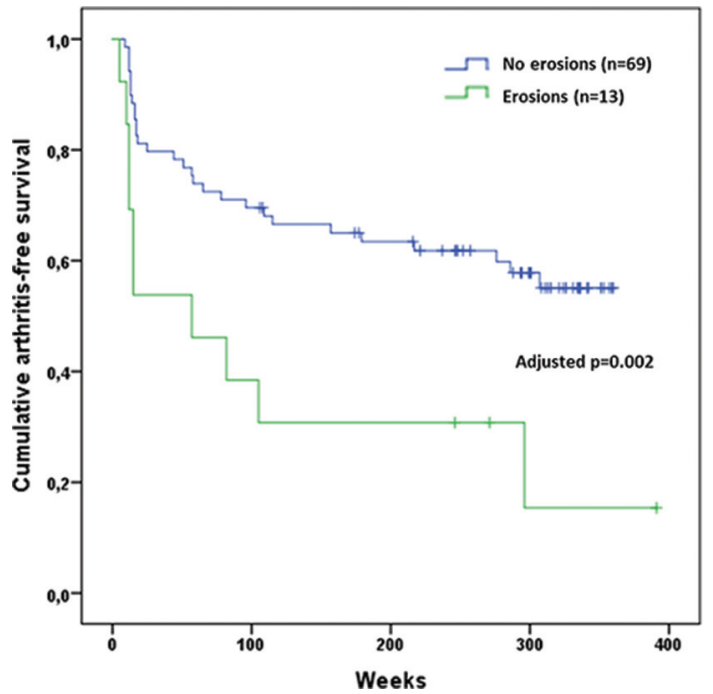

Figure 1. Survival plot illustrating progression to arthritis during follow-up in relation to the presence of ultrasound erosions at baseline among patients with anti-citrullinated protein antibodies and musculoskeletal pain.

Disclosure of Interests: Michael Ziegelasch Consultant for: AbbVie, MSD, Pfizer, and BK-Medical, Emma Eloff: None declared, Hilde Berner Hammer Speakers bureau: speakers fee from AbbVie, Bristol-Myers

Squibb, Roche, UCB Pharma and Pfizer, Jan Cedergren: None declared, Klara Martinsson: None declared, Åsa Reckner: None declared, Thomas Skogh: None declared, Mattias Magnusson: None declared, Alf Kastbom Consultant for: Roche and Pfizer, Employee of: Sanofi, Speakers bureau: UCB and BMS DOI: 10.1136/annrheumdis-2019-eular.1594 